\title{
Arsenic-bridged magnetic interactions in an emerging 2D FeAs nanostructure on MnAs
}

\author{
Christian Helman, ${ }^{1,2, \text { f }}$ Valeria Ferrari, ${ }^{3,4}$ and Ana Maria Llois $^{3,4,5}$ \\ ${ }^{1}$ Gerencia de Tecnologías de la Información y las Comunicaciones- \\ Centro Atómico Constituyentes - (1650) San Martín - Argentina \\ ${ }^{2}$ Instituto Sábato - Universidad Nacional de San Martín - Argentina \\ ${ }^{3}$ Gerencia de Investigación y Aplicaciones - Centro Atómico Constituyentes - (1650) San Martín - Argentina \\ ${ }^{4}$ Consejo Nacional de Investigaciones Científicas y Técnicas, C1033AAJ, CABA, Argentina \\ ${ }^{5}$ Departamento de Física Juan José Giambiagi, FCEN, \\ Universidad de Buenos Aires, (1428) Buenos Aires, Argentina
}

(Dated: June 16, 2015)

\begin{abstract}
The extreme case of a Fe monolayer deposited onto a manganese arsenide (MnAs) substrate is analysed using density functional theory. We find that a FeAs quasi 2D antiferromagnetic surface nanostructure emerges. This nanostructure, which is magnetically nearly decoupled from the substrate, is due to bonding effects arising from the arsenic atoms bridging the Fe magnetic interactions. These interactions are studied and modeled using a Heisenberg-type Hamiltonian. They display an angular dependence which is characteristic of superexchange-like interactions, which are of the same order of magnitude as those appearing in Fe-based pnictides.
\end{abstract}

PACS numbers: 75.70.Ak, 75.30.Et, 75.10.Hk

Keywords: electronic structure, superexchange, DFT, Heisenberg model

\section{INTRODUCTION}

In the last decade, due to the technological importance in the realm of spintronic devices, there has been theoretical and experimental interest in the study of $\mathrm{Fe}$ films grown on metallic ${ }^{112}$ as well as on semiconducting substrates $\sqrt[314]{14}$ Among the metallic substrates of interest, work has been done on $\mathrm{Fe}$ monolayers grown on tungsten ${ }^{5}$ and iridium,$\frac{6}{6}$ and in the last years, much effort has been devoted to the properties of nanometer thick Fe epilayers epitaxially grown on $\mathrm{MnAs} / \mathrm{GaAs}(001) ! 7 \cdot 10$

MnAs is a magnetic metallic material with several polymorphic transformations as a function of temperature 1113 Interestingly, at low temperatures, when MnAs is in its ferromagnetic $\alpha$-phase, it has been observed that deposited Fe films are magnetically decoupled from the MnAs substrate.$^{7 / 8}$ This has led to the idea of taking advantage of the magnetic and structural phase transition of $\mathrm{MnAs}$, that happens near room temperature and use it to develop a magnetic template..$^{7 / 14 \mid 15}$

In recent works, $\frac{15 \mid 16}{16}$ ultrathin Fe films were grown on MnAs/GaAs(001) substrates proposing and experimentally exploring a possible application of these combined systems. The authors made use of a laser to locally induce a Fe magnetization switching, profiting on the temperature-driven phase transition of MnAs. $\frac{16}{16}$

Actually, the involved magnetic interactions at the interesting $\mathrm{Fe} / \mathrm{MnAs}$ interface remain, still, an open question. $\frac{16}{6}$ The purpose of the present work is, thereafter, to study the magnetic interactions at the early stage of Fe deposition on MnAs by analyzing the extreme case of a Fe monolayer (ML). This Fe coverage limit has not yet been approached, neither theoretically nor experimentally and we expect that our findings will open the way to further explore it. We consider that the MnAs substrate is in its $\alpha$-phase, which is the stable structure below room temperature.

We find that a 2D FeAs nanostructure forms at the surface under study. This happens if one considers the experimental growth conditions of the MnAs substrate, which involve an As-rich atmosphere rendering an As termination ${ }^{[17}$ By means of a Heisenberg-type Hamiltonian, we show that the magnetic couplings at this nanostructure and at the interface can be explained through a mediation mechanism led by the As atoms. The surface nanostructure displays a magnetic ground state completely different to the one of a thicker Fe overlayer. ${ }^{[18} \mathrm{It}$ is likely that the emerging physics at this early growth stage presents some kind of similarity to the one of FeAsbased materials, which have been and are widely studied in the last few years 1921

\section{CALCULATION DETAILS}

We simulate the $\alpha$-MnAs substrate with a supercell built by three MnAs unit cells separated by a vacuum region of $10 \AA .22$ The periodic slab electronic calculations are performed within the framework of ab initio density functional theory (DFT) as implemented in the VASP code ${ }^{23}$ PAW pseudopotentials are used, ${ }^{24}$ along with the generalized gradient approximation (GGA) to the exchange and correlation potential within the PBE parametrization. ${ }^{25}$ The kinetic energy cut off is set to 350 $\mathrm{eV}$ while a $20 \times 20 \times 1$ mesh in the reciprocal space is considered. Internal atomic coordinates are fully relaxed until forces are smaller than $0.04 \mathrm{eV} / \AA$.

We assume that $\alpha$-MnAs grows in the [1100] direction, therefore, the in-plane cell parameters of the slab are taken as $a=3.73 \AA$, and $b=5.69 \AA \AA^{22}$ No inver- 


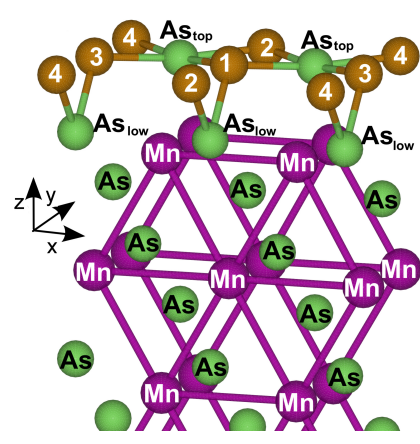

(a)

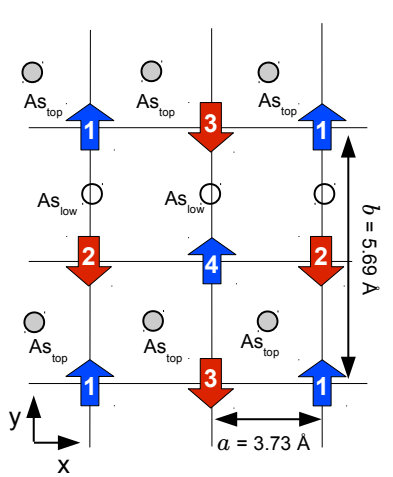

(b)
FIG. 1. (a) Relaxed structure of a Fe monolayer on the MnAs substrate. Green/violet/brown spheres stand for As/Mn/Fe ions, respectively. There are two types of As atoms at the interface, namely $\mathrm{As}_{\text {top }}$ (lying in the Fe plane) and As $\mathrm{s}_{\text {low }}$ located in-between the Fe and Mn planes. The topmost 2D nanostructure is composed of Fe and Astop atoms. (b) Schematic top view of the FeAs nanostructure displaying the ground state antiferromagnetic configuration (AFM-1). As low atoms are shown for reference. The up/down arrows denote parallel/antiparallel Fe magnetic moments with respect to the Mn ones. The numbers within the arrows refer to Fe atoms as in (a).

sion symmetry is imposed in order to have full MnAs formula units in the unit cell of the periodic slab, and consequently the two free surfaces of the slab show different atomic terminations, thus allowing to analyze the two possible interfaces between the Fe film and the MnAs substrate.

\section{INTERPLAY BETWEEN CRYSTAL STRUCTURE AND MAGNETISM}

We focus on the situation given by the experimental growth conditions of MnAs, which favor the As termination on top of which the Fe ML is assumed to be deposited 17 Fe-impurities in MnAs bulk occupy preferentially Mn-substitutional positions ${ }^{26 / 27}$ and therefore we propose that the interfacial Fe atoms follow the MnAs structure occupying $\mathrm{Mn}$ sites. After relaxation of the atomic coordinates, a sharp interface without any Fe interdiffusion is obtained, but an important reconstruction involving the As atoms occurs. The As-terminated surface presents two non equivalent atomic positions for the As atoms. One of the arsenic non-equivalent positions, denoted by $\mathrm{As}_{\text {low }}$, lies slightly above the last Mn plane of the substrate, while the other one, labeled as $\mathrm{As}_{t o p}$, ends up lying, after relaxation, close to the Fe plane rendering a quasi 2D nanostructure as shown in Fig. 1(a). It is worthwhile to remark that our calculations indicate that the Fe monolayer is stable for both terminations of the MnAs substrate.

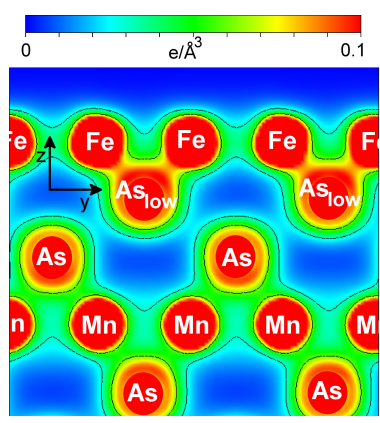

(a)

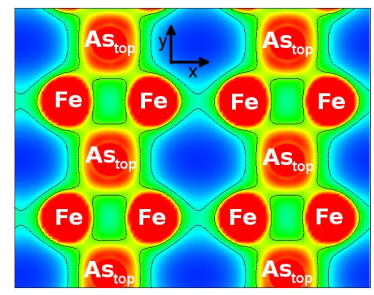

(c)

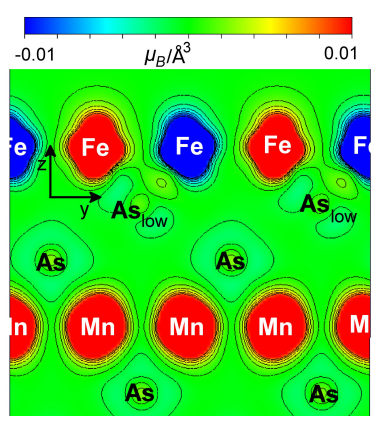

(b)

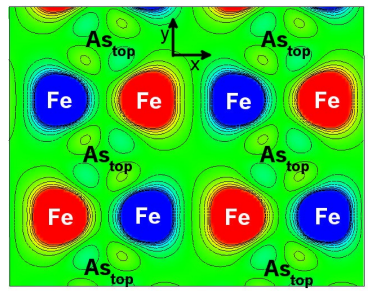

(d)
FIG. 2. Left/right columns correspond to charge/spin density maps. (a)-(b) zy cut containing As $_{\text {low }}$ atoms. (c)-(d) xy cut containing the FeAs nanostructure. See labels and coordinate system in Fig. 1.

Taking into account that crystal structure and magnetism influence each other, we propose different magnetic configurations (MCs) for the 2D nanostructure allowing the internal positions within the cell to relax in each case. In Table I] all the considered MCs are summarized along with the resulting Fe magnetic moments and the relative energies of the configurations. For different MCs, positive/negative Fe magnetic moments imply parallel/antiparallel alignment with respect to the Mn atoms of the substrate. These moments are denoted by $\uparrow / \downarrow$ in Table I. Note that the magnetic moments of the Fe atoms are close to their Fe bulk value $\left(\approx 2.22 \mu_{B}\right)$ despite being in a 2D nanostructure.

Fig[1 shows the relaxed atomic structure and a

TABLE I. Energy differences among the magnetic configurations considered. In the second column, the magnetic configurations are labelled with the spin orientation corresponding to the Fe atoms numbered in Fig. 11.a) following the order $|\mathbf{1 2 3 4}\rangle$. All the energies, $\Delta \mathrm{E}$, are referred to the AFM-1 ground state configuration. The Fe-magnetic moments (MM) are also given.

\begin{tabular}{ccccc} 
MC & $|\mathbf{1 2 3 4}\rangle$ & $\Delta E$ & $\operatorname{MM}(\uparrow)$ & $\operatorname{MM}(\downarrow)$ \\
& & {$[\mathrm{meV} /$ Fe ion $]$} & {$\left[\mu_{B}\right]$} & {$\left[\mu_{B}\right]$} \\
\hline AFM-1 & $|\uparrow \downarrow \downarrow \uparrow\rangle$ & 0.0 & 2.27 & -2.29 \\
AFM-2 & $|\downarrow \uparrow \downarrow \uparrow\rangle$ & 48.1 & 2.22 & -2.15 \\
AP & $|\downarrow \downarrow \downarrow \downarrow\rangle$ & 78.8 & & -2.20 \\
P & $|\uparrow \uparrow \uparrow \uparrow\rangle$ & 101.4 & 2.12 & \\
AFM-3 & $|\uparrow \uparrow \downarrow \downarrow\rangle$ & 102.3 & 2.10 & -2.25
\end{tabular}


schematic top view of the magnetic ground state denoted as AFM-1. To characterize AFM-1, we present in Fig 2 the charge and spin density distributions for different planes of the slab. It can be observed that beyond the uppermost As layer, the electronic distribution of the substrate is barely affected by the presence of the Fe overlayer. Figs 2(a) and (c) show that Fe and As atoms are covalently bond, and this covalency is noticeably stronger than the one between $\mathrm{Mn}$ and As atoms. The topmost Mn-layer relaxes slightly towards the developed Fe-As overlayer, and the only appreciable effect on the $\mathrm{Mn}$ atoms is a small deformation of the spin cloud due to the antiferromagnetic character of the Fe network (See Fig. 2(b)).

The spin densities shown in Fig 2(b) and (d), reveal a strong localization of the $\mathrm{Fe}$ atoms' magnetic moments. The magnetic role played by the bridging Asatoms can be recognized in the reconstructed surface layer. Although As low presents a small net magnetization $\left(<0.01 \mu_{B}\right)$, its lobes are antiferromagnetically aligned with the nearest $\mathrm{Fe}$ atoms.

The above-described features point towards the presence of a superexchange-like mechanism as the main contribution to the magnetic interactions among the $\mathrm{Fe}$ atoms at the nanostructure. 19

Actually, the magnetic interaction is highly dependent on the bonding distance, namely, the Fe-As ones within the nanostructure. To highlight this issue, we perform a simulation where the $\mathrm{Fe}$ atoms are rigidly moved away from the substrate. Fig. 3(a) shows that the AFM-1 configuration is the lowest energy one for separations $\Delta d$ of up to $0.5 \%$ of the equilibrium distance. For displacements beyond these values, the $\mathrm{P}$ and $\mathrm{AP}$ arrangements are the energetically preferred ones and are nearly degenerate. As denoted in Table I. P/AP configurations have the Fe spins aligned among them while being parallel/antiparallel aligned to the Mn atoms of the substrate. Therefore, the degeneracy of $\mathrm{P}$ and AP configurations indicates a small coupling between the Fe overlayer and the MnAs substrate for those displacements for which the Asbridging breaks down. Otherwise, in another simulation, the nanostructure as a whole (that is the Fe overlayer along with the Astop atoms) is rigidly separated from the substrate. The results are displayed in Fig 3(b). In this case, the AFM-1 configuration is always the preferred one, independently of the value of $\Delta d$ and, no degeneracy between $\mathrm{P}$ and $\mathrm{AP}$ is observed.

We conclude that the antiferromagnetic configuration AFM-1 is the lowest energy one, and is originated by the presence of the $\mathrm{As}_{t o p}$ atoms. This behaviour of the Fe monolayer is similar to what has already been reported for a monolayer of Fe grown on W(001), which at the equilibrium interlayer distance shows an AFM ground state. ${ }^{[2}$ As in our case it is the hybridization with the substrate what dominates the Fe-Fe magnetic interaction. Additionally, the slight preference for the AP configuration over the $\mathrm{P}$ one in Fig 3(b) indicates a weak antiferromagnetic coupling between the FeAs nanostruc-

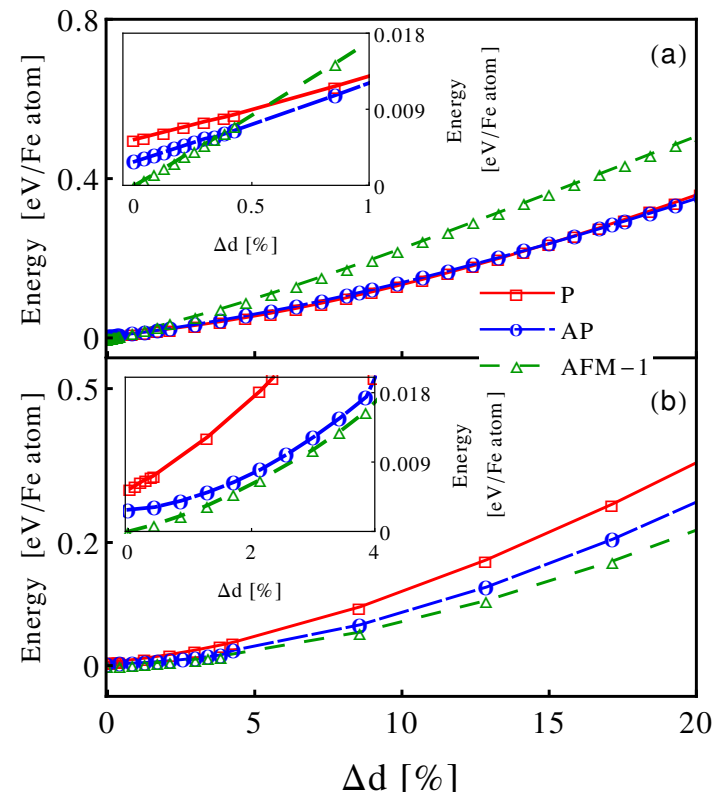

FIG. 3. Energy behavior of different magnetic configurations when (a) a Fe-only layer is rigidly separated from the substrate or when (b) the Fe-Astop layer (i.e. nanostructure) is separated from the substrate. $\Delta d$ gives the relative displacement with respect to the equilibrium distance for each magnetic configuration. Both insets are zoomed-in areas of the corresponding graphs. Green dashed line is the energy evolution of the AFM-1 and dashed blue/ red smooth line corresponds to the $\mathrm{AP} / \mathrm{P}$ configuration. The inset in (a) shows that for small separations from the equilibrium position, the AFM-1 configuration is no longer the ground state. On the contrary, when Fe and Astop atoms move rigidly together as in (b), the AFM-1 is always the ground state.

ture and the closest Mn layer. This weak interaction reveals the decoupling role played by the $\mathrm{As}_{\text {low }}$ atoms in the determination of the magnetic interactions among $\mathrm{Fe}$ and $\mathrm{Mn}$.

\section{MODELING THE MAGNETIC COUPLINGS}

The magnetic ground state of the emerging surface nanostructure is similar to the ones found in the iron-pnictides. ${ }^{19}$ In previous works ${ }^{19 / 20 \mid 28}$ for ironpnictides, it was proposed that the Fe-Fe interactions are modified by As mediation and that they depend on the angle formed by the different Fe-As bonds appearing in the structures. Actually, in these interactions all valence orbitals of the different atoms are involved and it is difficult to isolate the orbitals responsible for each Fe-Fe or Fe-Mn couplings ${ }^{[28}$ Thereafter, similar to what is done in ref. ${ }^{19}$ we consider average As-bridged interactions among atomic spin moments to model the magnetic couplings of the system. Direct Fe-Fe interactions are also taken into 


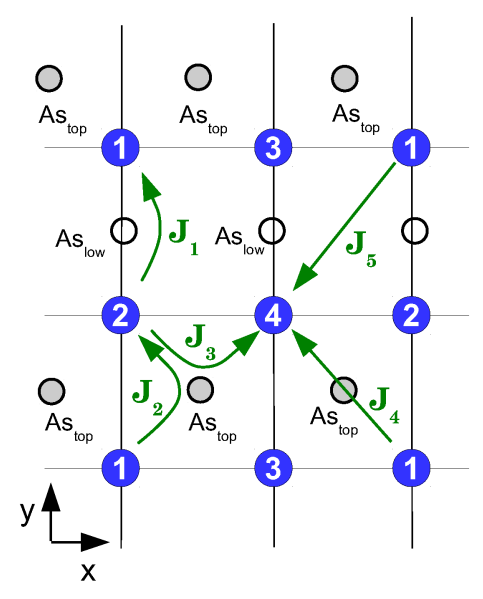

FIG. 4. Schematic picture of the magnetic couplings proposed within a Heisenberg model for the FeAs nanostructure that develops when a Fe monolayer is deposited on As terminated MnAs. The blue circles represent Fe atoms with their number in correspondence with Fig. 1(a). The model considers four different magnetic arsenic-mediated $\mathrm{Fe}-\mathrm{Fe}$ magnetic interactions, $J_{1}$ to $J_{4}$ sustaining different angles, and a long range Fe-Fe direct interaction, $J_{5}$. The Fe-Mn couplings $\left(J_{6}\right.$ and $J_{7}$ ) are also considered in our model, but are not shown in this picture.

account.

In order to estimate the values of the couplings, we use the atomic moment approximation with collinear configurations. Total energies obtained for different MCs within DFT are mapped onto a Heisenberg-type Hamiltonian. This Hamiltonian can be separated into two terms, $\hat{\mathbf{H}}_{0}$ that is independent of any MC configuration and, a second contribution that depends on spin operators $\hat{\mathbf{S}}$ and magnetic couplings $J_{i j}$. The spin operators are replaced by $\sqrt{s(s+1)} \sigma_{i}$, where $\sigma_{i}$ can take the values $+1(\uparrow)$ or $-1(\downarrow)$ denoting the magnetic moment orientation at site $i$. The proposed Hamiltonian is then given by,

$$
H=\hat{\mathbf{H}}_{0}-\sum_{i \neq j} J_{i j}\left(\sigma_{i} \cdot \sigma_{j}\right) .
$$

The values of the respective magnetic moments are included in the definition of $J_{i j}$, which can be positive/negative denoting ferromagnetic/antiferromagnetic interactions.

As a whole, we consider seven different $J_{i j}$ exchange parameters labelled as follows: $J_{1}$ to $J_{4}$ indicate Fe-Fe interactions mediated by an As atom for four different Fe-As-Fe angles, $\alpha . J_{5}$ denotes a direct Fe-Fe interaction, while $J_{6}$ and $J_{7}$ stand for Fe-Mn couplings mediated by As for different Fe-As-Mn angles, $\beta . J_{6}\left(J_{7}\right)$ represents an average Fe-Mn interaction for $\beta$ larger (smaller) than $90^{\circ}$. The couplings among the Fe atoms of the nanostructure are schematically depicted in Fig 4 . The values of the different $J_{i}$ are obtained through a least-square fitting procedure ${ }^{29}$ over ten different MCs, namely 8 an-
TABLE II. Magnetic spin couplings corresponding to the interactions depicted in Fig $4 \alpha$ is the Fe-As-Fe angle and $\beta$ is the Fe-As-Mn one.

\begin{tabular}{cccccc}
$\mathrm{J}$ & mediated by & atoms & Angle & \multicolumn{2}{c}{ distance $\mathrm{meV} /$ atom } \\
\hline$J_{1}$ & As-low & $\widehat{1,2}$ & $\alpha=73^{\circ}$ & $2.89 \AA$ & -21.2 \\
\hline$J_{2}$ & & $\widehat{1,2}$ & $\alpha=79^{\circ}$ & $2.8 \AA$ & -31.5 \\
$J_{3}$ & As-top & $\widehat{1,3}$ & $\alpha=106^{\circ}$ & $3.73 \AA$ & -36.7 \\
$J_{4}$ & & $\widehat{1,4}$ & $\alpha=180^{\circ}$ & $4.66 \AA$ & 4.4 \\
\hline$J_{5}$ & direct & & & $4.72 \AA$ & -4.3 \\
$J_{6}^{F e, M n}$ & both As & & $\beta>90^{\circ}$ & & -6.7 \\
$J_{7}^{F e, M n}$ & both As & & $\beta<90^{\circ}$ & & 3.1
\end{tabular}

tiferromagnetic (AFM) arrangements and also the AP and $\mathrm{P}$ configurations.

The resulting magnetic couplings, appearing in Table II, reveal an interesting angular dependence. In the case of the As-bridged Fe-Fe interactions, the magnetic coupling is increasingly antiferromagnetic in the angular range $73^{\circ}<\alpha<106^{\circ}\left(J_{1-3}\right)$, while for $\alpha \approx 180^{\circ}\left(J_{4}\right)$ the coupling is ferromagnetic. The As-mediated Fe-Mn interactions are antiferromagnetic for $\beta<90^{\circ}$ and ferromagnetic otherwise $\left(J_{6}\right.$ and $\left.J_{7}\right)$.

We try to understand the above angular dependence by means of Goodenoughs' rules ${ }^{30}$ by isolating the three atoms involved in each As-bridged interaction. For this purpose, Fe-As-Fe and Fe-As-Mn triatomic structures are considered. We perform simulations changing the angles $\alpha$ and $\beta$ held by these structures while fixing the As$\mathrm{Fe}$ and As-Mn distances to the values they attain after relaxation in the $\mathrm{Fe} / \mathrm{MnAs}$ heterostructure. Within this structure, both FM and AFM alignments are proposed. In Fig 5 (a) and (b), the energy differences between AFM and FM configurations as a function of $\alpha / \beta$ angles are shown.

The Fe-As-Fe triatomic structure presents two magnetic transitions, one at $72^{\circ}$ when the magnetic ground state changes from FM to AFM and, the other one at $150^{\circ}$ when the magnetic state turns FM again. Prior to the first transition, the FM alignment is actually due to a direct interaction between the two Fe atoms, because their distance is less than the nearest neighbour distance in bulk Fe. In this small angle region, the charge isosurface shown in Fig. 5(a), reveals this direct Fe-Fe interaction. Increasing the $\alpha$ angle, the Fe-Fe coupling starts being mediated by As and the triatomic structure turns AFM. As it can be seen in Fig 5 (a), between $72^{\circ}$ and $150^{\circ}$ the lowest energy configuration is antiferromagnetic. According to Goodenough' rules, this is due to the fact that the As- $p_{\pi}$ orbital hybridizes with the Fe- $d_{y z}$ and Fe- $d_{x z}$ ones, while the As- $p_{\sigma}$ orbital mainly hybridizes with Fe$d_{x^{2}-y^{2}}$.

In the extreme limit case of $180^{\circ}$, the interacting Asorbitals are mainly the $p_{z}$ and $p_{y}$ ones while the bonding direction is along the $\hat{x}$-axis. These two orbitals are degenerate, hybridize with the Fe- $d_{x z}$ and Fe- $d_{x y}$ ones and are perpendicular to the plane of the Fe-As-Fe structure. 


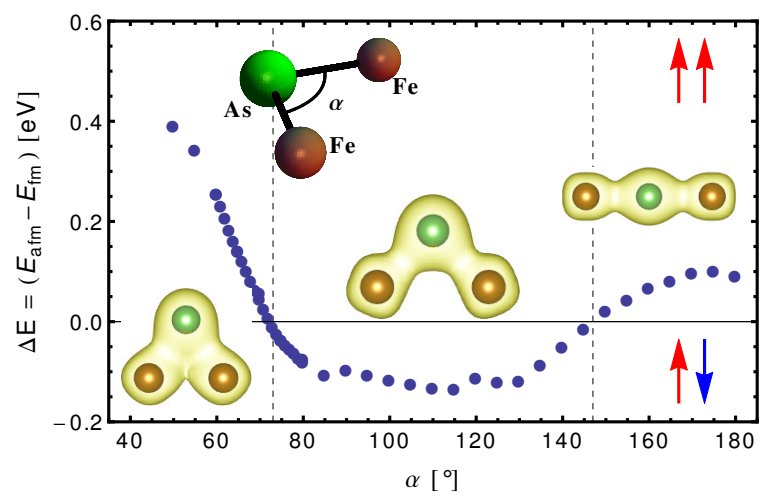

(a)

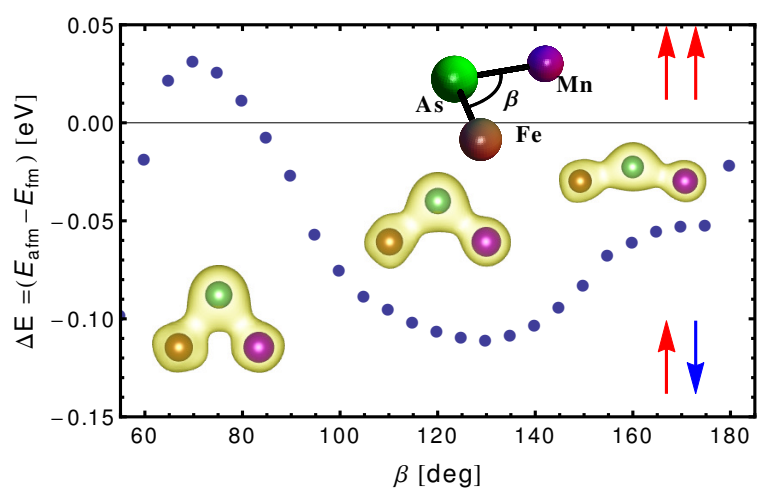

(b)

FIG. 5. Energy difference between antiferromagnetic and ferromagnetic configurations for simulated (a) Fe-As-Fe and (b) Fe-As-Mn structures, as a function of the corresponding angle $\alpha / \beta$. Positive/negative values indicate ferromagnetic/antiferromagnetic coupling. In (a) two magnetic transitions are observed when $\alpha$ increases, one from ferromagnetic to antiferromagnetic at $72^{\circ}$ and viceversa at $150^{\circ}$. In (b) there is only one transition at $85^{\circ}$. A typical charge isosurface graph is superimposed at each stage.

The magnetic interaction mediated by these orbitals is in fact FM and can also be understood with Goodenoughs' rules. For $\alpha$ between $140^{\circ}$ and $180^{\circ}$, there is a competition between the AFM and the FM contributions, which gradually change their relative weight until the maximum ferromagnetic contribution is attained at $180^{\circ}$.

In Fig $5(\mathrm{~b})$ we focus on the Fe-As-Mn triatomic system. In this case only one magnetic transition, from FM to AFM, is observed at $\beta=90^{\circ}$. The antiferromagnetic coupling has the same origin as the one present in the Fe-As-Fe structure discussed above. The net coupling between the nanostructure and the substrate is small as compared to the net antiferromagnetic interactions within the nanostructure, pointing towards a nearly magnetically decoupled overlayer.

Going back to the 2D nanostructure, the bonding angles related to $J_{1}-J_{3}$ which are antiferromagnetic, fall within the antiferromagnetic angular range obtained for the Fe-As-Fe structure, while the angle associated to the ferromagnetic $J_{4}$ coupling lies in the ferromagnetic an- gular range of the Fe-As-Fe one. For the As-mediated couplings between $\mathrm{Mn}$ and $\mathrm{Fe}$ atoms in the nanostructure, the behavior given by the signs of $J_{6}(\beta>90)$ and $J_{7}(\beta<90)$ is also consistent with the antiferromagnetic/ferromagnetic trends obtained for the triatomic FeAs-Mn structure in the corresponding angular range.

The fact that the results for the triatomic structures correlate so well with the parametrized Heisenberg Hamiltonian, points towards a short range interactions' scenario.

Taking the above into account, the As-bridged interatomic magnetic interactions, which define the magnetic ground state of the $2 \mathrm{D}$ metallic nanostructure can be traced back to superexchange-like interactions within triatomic units.

\section{FINAL REMARKS}

In this work we have analyzed the reconstruction and magnetic interactions that are expected to take place after deposition of $\mathrm{Fe}$ on a MnAs thin film grown on $\mathrm{GaAs}(001)$ at the early growth stage in the Fe monolayer range. In this limit, an antiferromagnetic surface nanostructure composed of $\mathrm{Fe}$ and As atoms emerges, in such a way that Fe-Fe magnetic interactions are mainly arsenic mediated, building Fe-As-Fe bridges. The magnetic structure of this overlayer, could in principle be observed using spin-polarized tunneling microscopy ${ }^{[2]}$ It is interesting to remark that the As-atoms at the interface provide simultaneously the magnetic interaction among Fe atoms as well as a decoupling mechanism of the overlayer from the MnAs substrate. The magnetic interaction with the substrate is weak as compared to the in-plane ones. The net exchange coupling among the interface Fe and Mn atoms, around $3.8 \mathrm{meV}$ (100 Oe), is within the range expected for the effective exchange field, $H_{e x}$, as given in ref. 9

Through the mapping of $a b$ initio total energy results onto a Heisenberg type Hamiltonian, we are able to characterize the As-mediated Fe-Fe magnetic interactions. The As bridge plays a crucial role in the determination of the magnetic ground state of the resulting heterostructure, in a similar way to what happens in the Fe pnictides superconductors ${ }^{19}$ Most of the couplings obtained for the mediated Fe-Fe interactions are of the same order of magnitude as those of the mentioned iron pnictides, that is, around $50 \mathrm{meV} ! 19$ In the present case, we also consider Fe-Fe direct interactions, which are one order of magnitude smaller than the As-mediated one and similar to the As mediated Fe-Mn interactions across the interface. The sign of these superexchange-like magnetic couplings are interpreted resorting to triatomic Fe-As-Fe and Fe-As-Mn structures where Goodenoughs' rules are easily identified.

The appeared 2D FeAs nanostructure presents similarities to the FeSe monolayer recently grown on $\mathrm{SrTiO}_{3}{ }_{4}$ Therefore, our emerging system could be superconduct- 
ing upon proper doping or treatment. A thorough analysis of this possibility is out of the scope of this paper.

To sum up, when depositing Fe on MnAs, at the early monolayer stage of growth, a FeAs antiferromagnetic surface nanostructure develops, which is magnetically nearly decoupled from the MnAs substrate and constitutes a $2 \mathrm{D}$ object that poses challenges and opportunities for heterostructure-based interface engineering.

\section{ACKNOWLEDGMENTS}

We thank L.Steren, M. Marangolo, J.Milano and V. Vildosola for helpful discussions. C.H., V.F and A.M.L. belong to the Institute of Nanoscience and Nanotechnology (INN) of the Atomic Energy Agency (CNEA), Argentina and to LIFAN (Laboratorio Internacional Franco-Argentino en Nanociencias). They acknowledge financial support from ANPCyT (PICT-20111187 and 1857), CONICET (PIP00273) and UBACYTW354 (UBA).
* helman@tandar.cnea.gov.ar

1 B. Hardrat, A. Al-Zubi, P. Ferriani, S. Blügel, G. Bihlmayer, and S. Heinze, Phys. Rev. B 79, 094411 (2009)

${ }^{2}$ A. Kubetzka, P. Ferriani, M. Bode, S. Heinze, G. Bihlmayer, K. von Bergmann, O. Pietzsch, S. Blügel, and R. Wiesendanger, Phys. Rev. Lett. 94, 087204 (2005)

${ }^{3}$ S. Mirbt, B. Sanyal, C. Isheden, and B. Johansson, Phys. Rev. B 67, 155421 (2003).

${ }^{4}$ Sun Yi, Zhang Wenhao, Xing Ying, Li Fangsen, Zhao Yanfei, Xia Zhengcai, Wang Lili, Ma Xucun, Xue Qi-Kun, and Wang Jian, Sci. Rep. 4, 6040 (2014) Ge Jian-Feng, Liu Zhi-Long, Liu Canhua, Gao Chun-Lei, Qian Dong, Xue Qi-Kun, Liu Ying, and Jia Jin-Feng, Nat Mater 14, 285 (2015)

B. A. Hamad and M. Richter, Phys. Rev. B 83, 245135 (2011)

${ }^{6}$ Z. Tian, D. Sander, and J. Kirschner, Phys. Rev. B 79, $024432(2009)$.

' R. Breitwieser, M. Marangolo, J. Luning, N. Jaouen, L. Joly, M. Eddrief, V. H. Etgens, and M. Sacchi, Applied Physics Letters 93, 122508 (2008)

$\checkmark$ C. Helman, J. Milano, S. Tacchi, M. Madami, G. Carlotti, G. Gubbiotti, G. Alejandro, M. Marangolo, D. Demaille, V. H. Etgens, and M. G. Pini, Phys. Rev. B 82, 094423 (2010)

${ }^{9}$ M. Sacchi, M. Marangolo, C. Spezzani, L. Coelho, R. Breitwieser, J. Milano, and V. H. Etgens, Phys. Rev. B 77, $165317(2008)$

io S. Tacchi, M. Madami, G. Carlotti, G. Gubbiotti, M. Marangolo, J. Milano, R. Breitwieser, V. H. Etgens, R. L. Stamps, and M. G. Pini, Phys. Rev. B 80, 155427 (2009)

${ }^{i 1}$ N. Mattoso, M. Eddrief, J. Varalda, A. Ouerghi, D. Demaille, V. H. Etgens, and Y. Garreau, Phys. Rev. B 70, 115324 (2004)

12 A. K. Das, C. Pampuch, A. Ney, T. Hesjedal, L. Däweritz, R. Koch, and K. H. Ploog, Phys. Rev. Lett. 91, 087203 (2003)

${ }^{13}$ L. B. Steren, J. Milano, V. Garcia, M. Marangolo, M. Eddrief, and V. H. Etgens, Phys. Rev. B 74, 144402 (2006).

14 M. Sacchi, M. Marangolo, C. Spezzani, R. Breitwieser,
H. Popescu, R. Dealaunay, B. Rache Salles, M. Eddrief, and V. H. Etgens, Phys. Rev. B 81, 220401 (2010).

15 Spezzani Carlo, Vidal Franck, Delaunay Renaud, Eddrief Mahmoud, Marangolo Massimiliano, Etgens Victor H., Popescu Horia, and Sacchi Maurizio, Sci. Rep. 5 (2015), $10.1038 /$ srep08120

${ }^{10}$ C. Spezzani, E. Ferrari, E. Allaria, F. Vidal, A. Ciavardini, R. Delaunay, F. Capotondi, E. Pedersoli, M. Coreno, C. Svetina, L. Raimondi, M. Zangrando, R. Ivanov, I. Nikolov, A. Demidovich, M. B. Danailov, H. Popescu, M. Eddrief, G. De Ninno, M. Kiskinova, and M. Sacchi, Phys. Rev. Lett. 113, 247202 (2014).

17 R. Breitwieser, F. Vidal, I. L. Graff, M. Marangolo, M. Eddrief, J.-C. Boulliard, and V. H. Etgens, Phys. Rev. B 80, 045403 (2009).

18 C. Helman, V. Ferrari, and A. M. Llois, Journal of Physics: Conference Series 568, 042011 (2014)

${ }^{19}$ F. Ma, Z.-Y. Lu, and T. Xiang, Phys. Rev. B 78, 224517 (2008)

${ }^{20}$ M. J. Calderón, B. Valenzuela, and E. Bascones, Phys. Rev. B 80, 094531 (2009).

${ }^{21}$ M. Aichhorn, L. Pourovskii, V. Vildosola, M. Ferrero, O. Parcollet, T. Miyake, A. Georges, and S. Biermann, Phys. Rev. B 80, 085101 (2009).

22 I. Rungger and S. Sanvito, Phys. Rev. B 74, 024429 (2006).

${ }^{23}$ G. Kresse and J. Furthmüller, Computational Materials Science 6, 15 (1996).

${ }^{24}$ G. Kresse and D. Joubert, Phys. Rev. B 59, 1758 (1999).

25 J. P. Perdew, K. Burke, and M. Ernzerhof, Phys. Rev. Lett. 77, 3865 (1996).

${ }^{26}$ H. Fjellvåg, A. Kjekshus, A. Andresen, and A. Ziba, Journal of Magnetism and Magnetic Materials 73, 318 (1988).

27 A. de Campos, D. L. Rocco, A. M. G. Carvalho, L. Caron, A. A. Coelho, S. Gama, L. M. da Silva, F. C. G. Gandra, A. O. dos Santos, L. P. Cardoso, P. J. von Ranke, and N. A. de Oliveira, Nat Mater 5, 802 (2006).

28 S. Graser, T. A. Maier, P. J. Hirschfeld, and D. J. Scalapino, New Journal of Physics 11, 025016 (2009).

29 A. Saúl and G. Radtke, Phys. Rev. Lett. 106, 177203 (2011)

so J. Goodenough, Phys. Rev. 100, 564 (1955) 